\title{
A HIGH RESOLUTION WIRE SCANNER FOR MICRON-SIZE PROFILE MEASUREMENTS AT THE SLC *
}

\author{
R. FULTON, J. HAGGERTY, R. JARED, R. JONES and J. KADYK \\ Lawrence Berkeley Laboratory, Berkeley, CA 94720, USA \\ C. FIELD and W. KOZANECKI \\ Stanford Linear Accelerator Center, Stanford University, Stanford, CA 94309, USA
}

\section{W. KOSKA}

University of Michigan, Ann Arbor, MI 48109, USA

Received 27 June 1988

Fine conductive fibers have been used to measure transverse beam dimensions of a few microns at the Stanford Linear Collider (SLC). The beam profile is obtained by scanning a fiber across the beam in steps as small as $1 \mu \mathrm{m}$, and recording the secondary emission signal at each step, using a charge sensitive amplifier. We first outline the mechanical construction and the analogue electronics of the wire scanner. We then describe its performance in test beams and in actual operation. The article closes with a brief discussion of performance limitations of such a beam profile monitor.

\section{Introduction}

The tuneup of the Stanford Linear Collider (SLC) [1] presents a challenge to measure beam sizes on a scale not previously encountered in high energy physics. The goal is to achieve bunches of electrons and positrons with approximate rms dimensions of $2 \mu \mathrm{m}$ transversely and $1 \mathrm{~mm}$ longitudinally. Collisions of beam bunches with such small dimensions have never before been attempted, and commonly used techniques (such as phosphor screen monitors) have a much too coarse resolution.

The device described here is a wire scanner that uses a very thin carbon fiber. This fiber is moved in uniform steps across the beam path while measuring the electrical signal generated by secondary emission [2]. Wire diameters of approximately 7 and $25 \mu \mathrm{m}$ have been used in SLC beams. Although this technique has been used at several high energy accelerators [3-8], we are not aware of its previous use at such a small scale of dimensions. Both the beam profile and the beam position are accurately determined by digitizing the wire position in steps as small as $1 \mu \mathrm{m}$, while recording an

\footnotetext{
* Work supported by the Department of Energy, contracts DE-AC03-76SF00515, DE-AC03-76SF00098 and DE-AC0276ER01112.
}

electrical signal whose amplitude is proportional to the amount of beam striking the wire. Other detection methods have been considered [3,4], such as the measurement of scattered beam particles or change in resistance due to temperature rise in the fiber, but - at these beam energies and repetition rates - secondary emission appeared to be the simplest method. Some new questions arise regarding the viability of the wire scanner in very intense beams. The SLC design calls for $5 \times 10^{10}$ electrons or positrons in a beam bunch of the very small dimensions given above. The heating which results from impact of such intense bunches may cause damage to or break the fiber. Carbon was chosen as the fiber material because the energy loss per unit length due to a crossing beam particle is relatively small, the melting (or sublimation) point is quite high, and the mechanical properties are otherwise good. The results described here were obtained in the tuneup phase of the SLC with the beam intensity well below its design value, so viability of the fibers has not been put to a significant test.

\section{Mechanical design}

Fig. 1 shows the SLC layout in plan view, with the wire scanner locations denoted. On each SLC machine pulse, electron and positron beam bunches travel the full length of the Stanford linac, about $3000 \mathrm{~m}$, gaining 


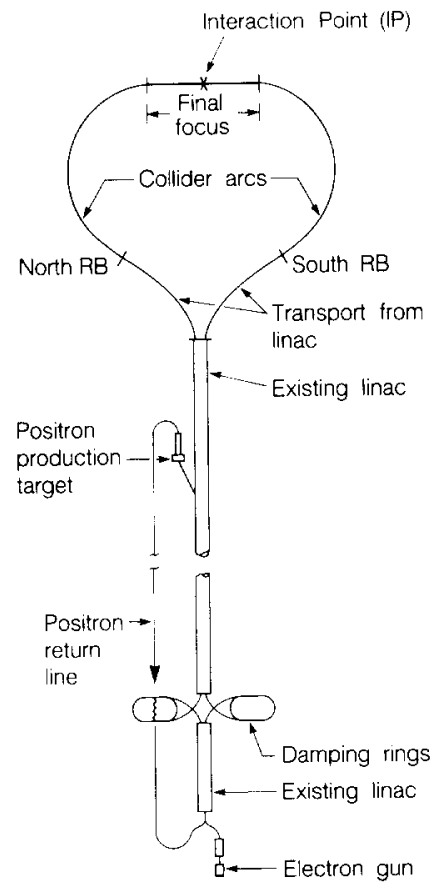

Fig. 1. Layout of Stanford Linear Collider (SLC). Wire scanners were built for the interaction point (IP), and the north and south reverse bend (RB) locations.

an energy of about $50 \mathrm{GeV}$ per particle. The design intensity is $5 \times 10^{10}$ particles per bunch, but in the tuneup phase, intensities of about $3 \times 10^{9}$ were typical. At the end of the linac the transverse radius of each bunch is about $90 \mu \mathrm{m}$, and it is here that the electron and positron bunches are separated by a magnet; then each is transported about $1300 \mathrm{~m}$ around an "arc" section to the final focus section. In the final focus the beam size is reduced to its minimum value at the collision, or interaction point (IP), of the electron and positron bunches.

Here, in a vacuum of about $10^{-9}$ Torr, the final focus wire scanners must operate. At the IP, three wire scanners were used, each having a measuring head with two carbon fibers of different diameters: about $7 \mu \mathrm{m}$ and $25 \mu \mathrm{m}$. Each wire scanner measured profiles in one of the three transverse directions: $X$ (horizontal), $Y$ (vertical) and $V$ (at $45^{\circ}$ to $X$ and $Y$ ). Two of the three measuring heads ( $X$ and $Y$ ) could be inserted into the beam simultaneously, by virtue of a $1 \mathrm{~mm}$ offset in position along the beam direction $(Z)$. A drawing showing the arrangement of the three wire scanners on their support plate is shown in fig. 2. A photograph of a measuring head is shown in fig. 3 , with wires installed. The carbon fibers, which are very brittle, were drawn around a curved surface of radius $4.5 \mathrm{~mm}$ on the ceramic holder to minimize the possibility of breakage. They are not mounted in a single plane normal to the beam axis, but are offset in $Z$ by about $350 \mu \mathrm{m}$, and spaced laterally (as seen by the beam) by $200 \mu \mathrm{m}$. The surface of the ceramic holder has been rendered slightly conductive by rubbing graphite on it; this prevents buildup of charge on the surface. Leadout wires were kept as short and as nearly perpendicular to the beam direction as possible to minimize the beam induction signal. These leads were insulated with ceramic beads, since commonly used (e.g., organic) insulators are unsuitable in a high vacuum environment and are also quite vulnerable to radiation damage.

The measuring head is attached to a support shaft, and leads from each end of the fiber are brought to feedthrough insulators in a housing mounted on the actuating stage of a commercially available linear positioning device [9]; the stage is moved by a stepping motor in $1 \mu \mathrm{m}$ steps. The vacuum seal is made by ceramic insulated feed throughs (BNC) welded into the wall of the housing, which is in turn coupled to the vacuum pipe using a bellows of nominal 3 in length, allowing a total actuator motion of about $30 \mathrm{~mm}$. Lead weights counterbalance the $5 \mathrm{~kg}$ vacuum loading on the bellows. The maximum residual loading of $3.2 \mathrm{~kg}$, due only to the bellows spring constant, is well within the manufacturer's $5 \mathrm{~kg}$ rating. Microswitches define the IN and RETRACTED positions, and interlock the stepping motor controllers. The normal running speed is 0.5 $\mathrm{mm} / \mathrm{s}$, corresponding to a $500 \mathrm{~Hz}$ stepping rate. About $45 \mathrm{~s}$ are required to insert the device to a PARK position close to the beam from which the actual scan is initiated. A typical beam scan takes about 15-20 s. The entire system is completely under computer control and may be operated from the Main Control Room by the SLC operators, or from any of several satellite locations.

Another version of the wire scanner has been built for the reverse bend (RB) locations in the SLC arcs (fig. 1). At each of these locations the beams are nominally circular with about $100 \mu \mathrm{m}$ radius. Focusing on the wire in either the $X$ or the $Y$ plane is expected to reduce the size to about $12 \mu \mathrm{m}$, and from this size the beam emittance can be determined. Moreover, verification can be made of the alignment of the beam optical axes with the $X$ and $Y$ planes, as well as the absence of any $X-Y$ correlation. The design allowing this additional measurement capability, not present in the final focus unit, is illustrated in fig. 4. The measuring head in this case supports the two wires of $25 \mu \mathrm{m}$ diameter with a calibrated spacing $(\sim 200 \mu \mathrm{m})$. While in the case of the final focus wire scanners the normal operation consists of mechanically scanning the wire past the beam, the measurement with the RB wire scanners is performed by deflecting the beam in stepwise increments past the wire, using small steering magnets. The stepping motor drive is used to insert and retract the pair of wires, and to rotate them about the beam axis after the measuring 


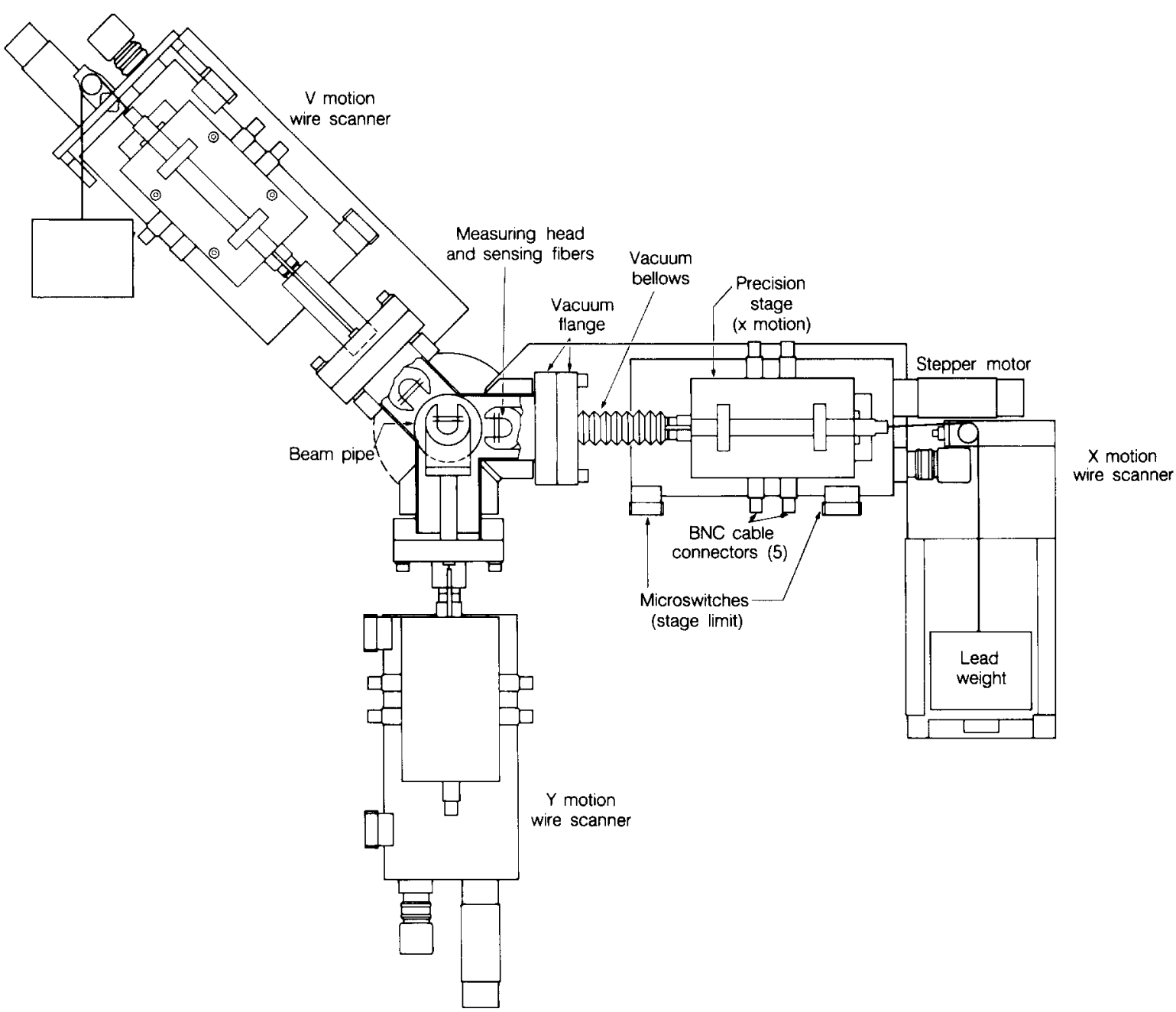

Fig. 2. Schematic of the arrangement of the three wire scanners mounted at the IP location. Some components are illustrated for the $X$-motion scanner.

head reaches the fully inserted position, as indicated by an electrical contact. The gear and ratchet mechanism which accomplishes this is seen in fig. 4. The calibrated wire spacing allows a direct calibration of the deflection magnet sensitivity, setting the scale for profile measurements. The wire rotation allows the beam waist measurement to be made in the directions of the $X$ or $Y$ axes, or any intermediate direction if $X-Y$ correlations are present.

\section{Electrical and electronic design}

\subsection{Preamplifier and CAMAC module}

Signals from each of the scanner wires are taken via $3 \mathrm{~m}$ lengths of RG223 cable (double-shielded) to a preamplifier which is completely surrounded by an electrostatic enclosure. In addition, the preamplifier is con- tained inside a heavy lead shield which protects it from ionizing radiation. The circuit block diagram is shown in fig. 5. The input signal from the wire is buffered by a charge sensitive preamplifier with provisions for placing a bias voltage on the wire and injecting a charge for the purpose of calibration. The signal from the preamplifier is amplified and shaped with a peaking time of about 3 $\mu \mathrm{s}$. The output is sent via $125 \mathrm{~m}$ of shielded multiconductor twisted pair cable to a CAMAC module which serves as an interface to the rest of the digitization and control modules, having connections for: calibration, $\mathrm{dc}$ wire bias, stepper motor control, encoder readout and analogue wire signal output. This signal is digitized by a CAMAC analogue-to-digital converter (ADC).

\subsection{Signal response}

Fundamental to the approach is the response of the electronics to the two types of input signal as illustrated 


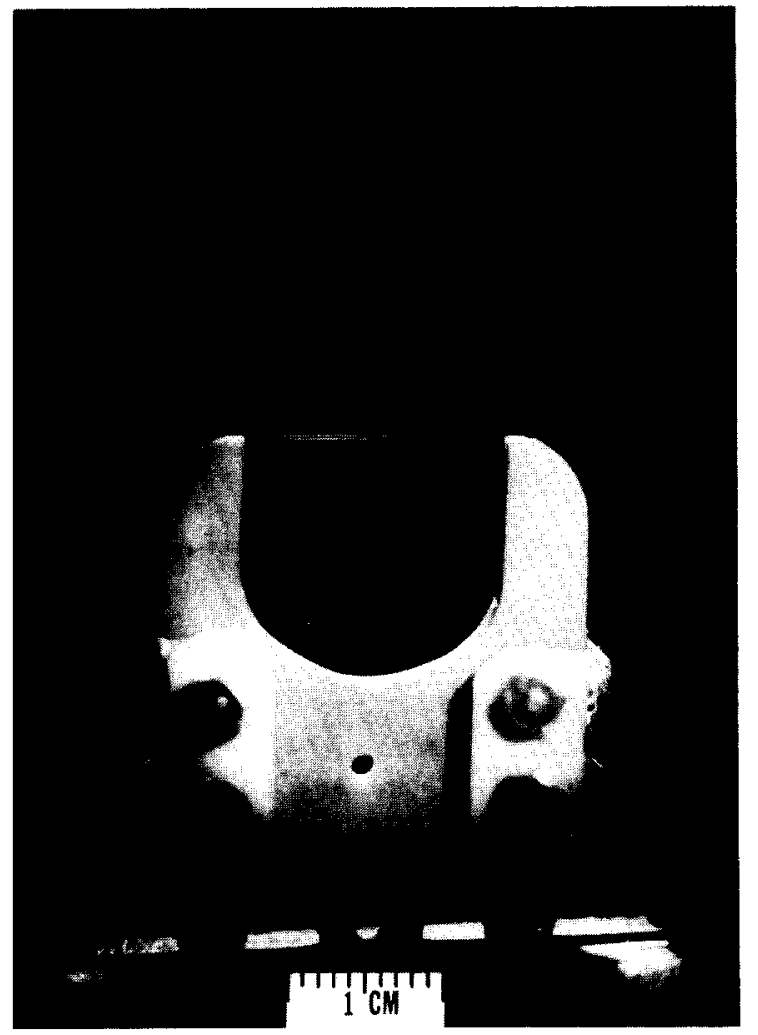

Fig. 3. Photograph of a measuring head used at the IP. Two measuring wires, $7 \mu \mathrm{m}$ and $25 \mu \mathrm{m}$ in diameter, are clearly visible.

in fig. 6. As the beam approaches the wire, a charge is induced that results in a current flow to the preamplifier; this occurs whether or not the beam strikes the wire. As the beam leaves the vicinity of the wire, the induced charge is removed, resulting in a current equal in magnitude, but opposite in direction to the first impulse. The separation in time of the two flows is of the order of a few picoseconds. The resulting shape (a differential of a Gaussian) due to the doublet input is shown in fig. 6 . There is an additional signal when the beam does strike the wire: the energy loss process in the wire results in the emission of secondary electrons. The resulting charge impulse is amplified to produce the Gaussian shape shown. To maintain the linear response of the preamplifier, essential to this approach, a $1 \mathrm{nF}$ shunt capacitor was added to attenuate the voltage extremes of the doublet input. In operation the analogue-to-digital converter samples the wave form at the crossover region of the doublet response. As is shown in the figures, this sample time will correspond to a maximum in the desired response to the secondary emission of electrons. The wire signal digitization is done in a LeCroy 2249 W ADC, gated by programmable gate delay and width units. The gate timing is provided by the SLC timing system. The dc response is a current of about $12 \mu \mathrm{A}$ to the ADC per $\mathrm{pC}$ of charge at the preamplifier input, or with a $0.5 \mu \mathrm{s}$ gate width, $6 \mathrm{pC}$ output to the ADC (channel 24). As an example, if the effective coefficient for secondary emission is about $2 \%$, and if $1 \times 10^{9}$ electrons (or positrons) struck the carbon fiber, then a $20 \mathrm{pC}$ charge would be digitized by the $\mathrm{ADC}$ (in channel 80).

\subsection{Position readout}

Position readout is provided by an incremental encoder on the stepper motor drive [9]. This encoder digitizes the optical signals produced as a circular grating attached to the motor drive rotates in front of a set of photocells. The encoder is sensitive to the direction of motion, and the entire system has a nominal accuracy of one step, or $1 \mu \mathrm{m}$. In addition, there is a linear potentiometer connected to the stage motion, having an accuracy of roughly $20 \mu \mathrm{m}$. This is primarily to provide a means of detecting possible malfunctions in the digital readout. Both of these readouts should agree with the number of steps requested by the command program.

\subsection{Calibration and fiber bias}

The electronic gain of the preamplifier can be determined by injection of a calibrated voltage pulse into the CAMAC module. There is also provision for introducing a negative dc bias voltage on the wire at this module, a technique which has been used to enhance the signal according to several published reports $[4,5,7]$. The basis of the effect is believed to be that some fraction of the very low energy electrons knocked out of the wire may return to the wire due to local electric fields, unless repelled by a small negative bias on the wire (usually $-30 \mathrm{~V}$ is sufficient). As will be described below, no effect due to the bias was found in tests with our wire scanner.

\section{Tests: problems and solutions}

\subsection{Bench test of beam induction effect}

As mentioned earlier, the response to a fast unipolar impulse (i.e., lasting $\ll 3 \mu$ s) is a bipolar pulse of $\sim 3$ $\mu \mathrm{s}$ zero-crossing time. The induction due to the field of the passing beam bunch, which has about a $1 \mathrm{~mm}$ length (rms), creates just such an impulse on the scan wire, lasting only about $10 \mathrm{ps}$. For a bunch close to the wire the amplitude of the induction signal can be many times the secondary emission signal. Due to its transient behavior, however, no signal from this effect should contribute to the measurement of the dc level at the 


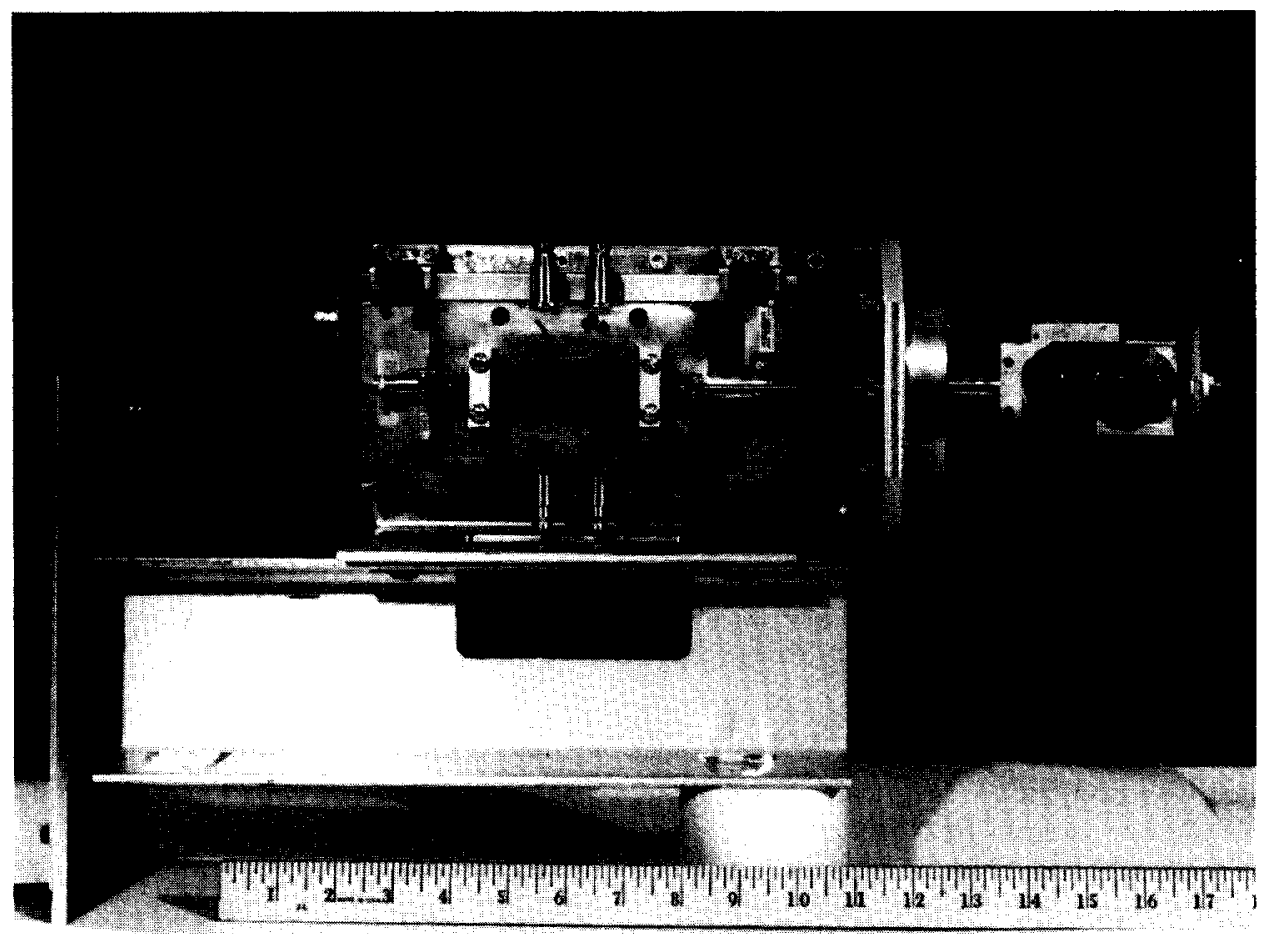

Fig. 4. Photograph of an RB wire scanner assembly. Evident are the components illustrated in fig. 2, as well as the ratchet and gear mechanism used to rotate the measuring head while in the "inserted" position.

sampling (crossover) time at $3 \mu \mathrm{s}$. Nevertheless, there was concern that some residual effects might perturb the desired signal (e.g., due to nonlinearities in the electronics system), since the induction amplitude could be much larger. Measurements were made of the beam induction pickup using a simulation of the beam bunch by a fast $(\sim 300 \mathrm{ps})$ pulse on a thin wire strung through the actual wire scanner housing. The actual preamplifier system was also used. The induction pulse was virtually unmeasurable at simulated beam levels of $\sim 10^{10}$ $\mathrm{e}^{-}$/pulse. When the scanning wire was oriented parallel to the beam, however, a distinctive induction signal was observed, at least 10 times larger than for the normal wire orientation. This is as expected, and led to careful placement of signal lead out wires as nearly perpendicu-

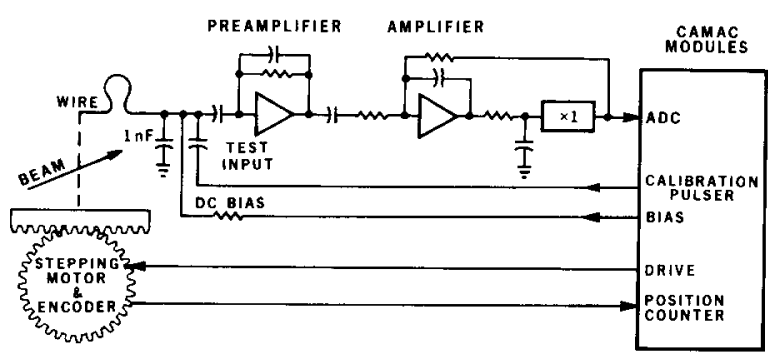

Fig. 5. Block diagram of electronics for wire scanners. lar to the beam axis as possible. Another feature is also advantageous: The induction signal is largely composed of very high frequency components, and that part generated at the fiber tends to be filtered out by the capacitances associated with the preamplifier input and the natural resistivity of the carbon fibers $(\sim 9 \mathrm{k} \Omega$ for the $2.6 \mathrm{~cm}$ length of $7 \mu \mathrm{m}$ fiber from the electrical connection to the center of the fiber).

\subsection{Beam tests}

Initial testing was performed in a $46 \mathrm{GeV}$ electron beam at the end of the linac, with intensities of about

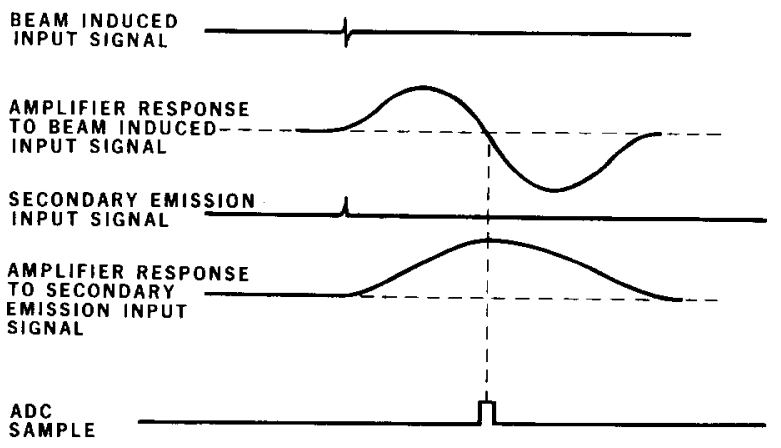

Fig. 6. Response of electronics to beam-induced signal and to the secondary emission signal. 

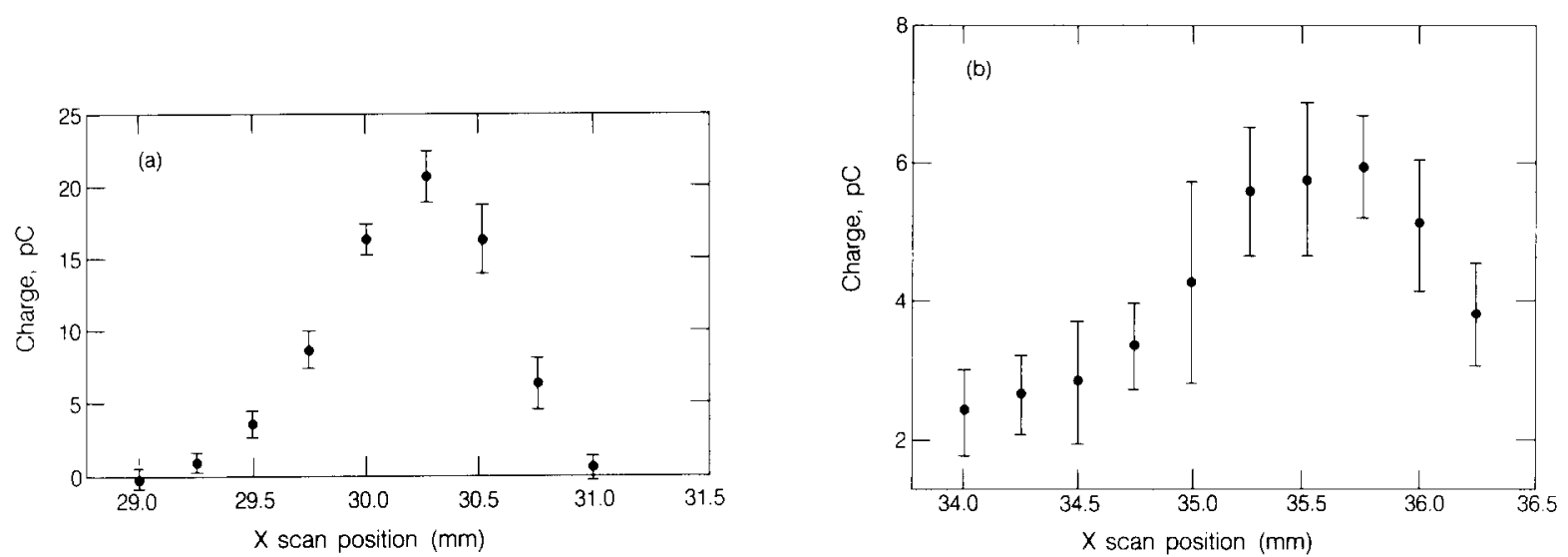

Fig. 7. First successful measurements of a beam profile performed at the end of the linac. (a) Profile taken with a $500 \mu \mathrm{m}$ diameter wire. (b) Profile taken with $\sim 150 \mu \mathrm{m}$ diameter wire. Both wires were made of copper, and the beam size was approximately $500 \mu \mathrm{m}$ rms, as determined by a scan using a phosphor screen.

$5 \times 10^{9} \mathrm{e}^{-} /$pulse and with 5 pulses $/ \mathrm{s}$. The drive system was controlled by a program running under BASIC on an IBM PC. Several crucial facts became apparent during these tests. Most importantly, this test area was not well shielded from stray particle fluxes, and also contained large amounts of electromagnetic radiation background, particularly close to the beam pipe. It was soon apparent that the preamplifier needed to be placed away from the beam pipe $(-2.5 \mathrm{~m})$ and inside a lead-brick house to shield against particle background. In addition, it was found necessary to have doubleshielded coax signal leads to protect against EM radiation. The combination of these improvements reduced the background level by a factor of 2000 . It was also

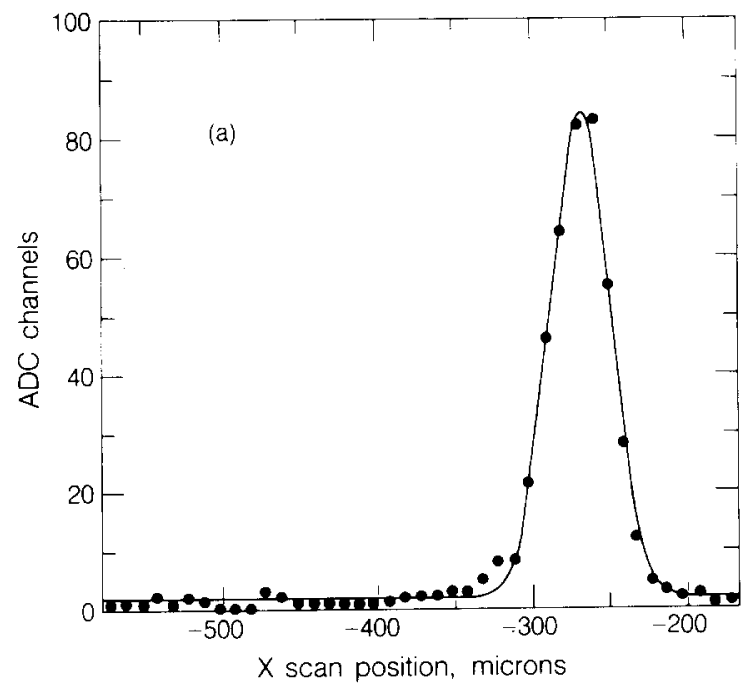

discovered that the signal cable must have solid-core insulation, as particle backgrounds produced gas ionization in the air-core cable used initially. It was verified by applying a bias voltage of about $100 \mathrm{~V}$, first with positive polarity, then negative, directly to the air core cable. There was a large background signal observed with either polarity, presumed to be due to collection of positive ions or electrons resulting from the ionization of the air core of the cable. In the case of solid insulation no such effect was observed. After these improvements a profile was easily measured using copper sensing wires, having diameters of $500 \mu \mathrm{m}$ and $150 \mu \mathrm{m}$, comparable with the measured beam size of $\sim 500 \mu \mathrm{m}$ rms (fig. 7).

Fig. 8. Beam profile measurement in $X$ direction at the IP. (a) Profile taken with $-25 \mu \mathrm{m}$ diameter fiber; (b) Profile with $7 \mu \mathrm{m}$ diameter fiber. Both fibers are carbon filaments. The $-200 \mu \mathrm{m}$ transverse spacing between the fibers is evident. The beam size is about $15 \mu \mathrm{m}$ rms. 


\section{Experience using wire scanners in the tuneup of SLC}

The wire scanner has been used to measure $X$ and $Y$ profiles of both $\mathrm{e}^{-}$and $\mathrm{e}^{+}$beams at the IP. Examples of typical profile plots are shown in figs. 8-10. Each point represents the measurement of a single pulse. The beam profile is obtained from a sequence of such measurements as the wire is scanned across the beam path. The vertical scale represents the wire signal response to a single SLC beam pulse, in ADC channel number, and the wire position is plotted in $\mu \mathrm{m}$ on the horizontal axis. The $\sim 200 \mu \mathrm{m}$ transverse spacing between the two wires is apparent in fig. 8, which shows the response from both wires on the same beam scan. In this case, the scan was done in the $X$ direction, and the resulting profile width is about $15 \mu \mathrm{m}$ rms. It will be noticed that the peak response of the large wire is about eight times that of the small wire, while the ratio of beam intensities subtended by the wire diameters is only about $3: 1$ for a Gaussian shape. This effect is currently being studied by investigation of the details of the SEM

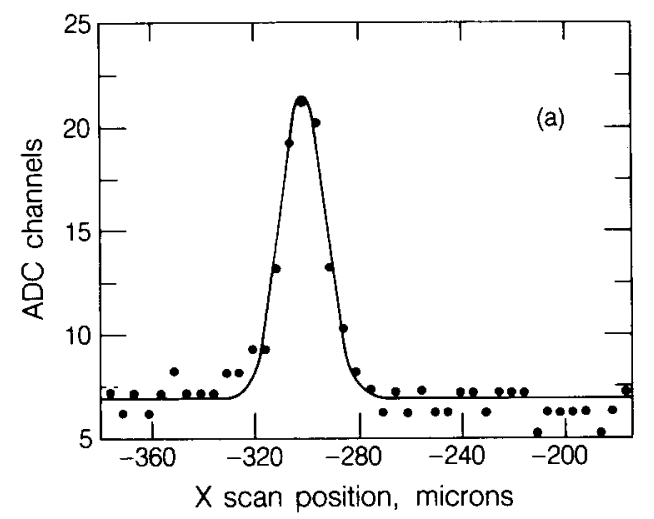

mechanism. Fig. 9 is an example of a measurement of the $X$ and $Y$ profiles on the small wire of an electron beam, taken less than $1 \mathrm{~min}$ apart, showing rms sizes 8.3 and $6.5 \mu \mathrm{m}$, respectively. The sizes quoted represent the widths of Gaussian distributions fitted to the raw data. They therefore are the convolution of the actual beam size, the pulse-to-pulse beam position jitter (if any), and the resolution effects due to the finite wire size. Fig. 10 shows $X$ and $Y$ scans of positrons and electron beams which were brought to the IP simultaneously, but which were not colliding at the time these scans were taken. This figure also clearly illustrates how the wire scanning technique can be used to bring the two beams into collision.

\section{Sense wire response}

\subsection{Secondary emission}

The principal mechanism of secondary emission produces soft electrons from "distant" collisions (i.e., large

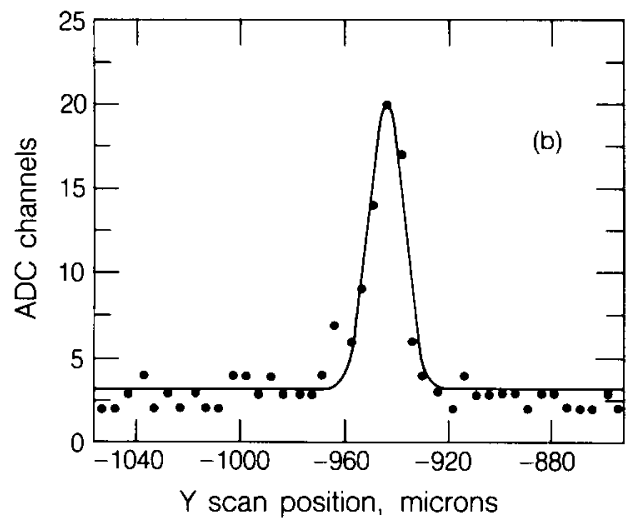

Fig. 9. Profile scans in $X$ and $Y$ directions of the beam taken less than $1 \mathrm{~min}$ apart using the smaller fiber. The beam size was $8.3 \mu \mathrm{m}$ in $X$ and $6.5 \mu \mathrm{m}$ in $Y, \mathrm{rms}$.
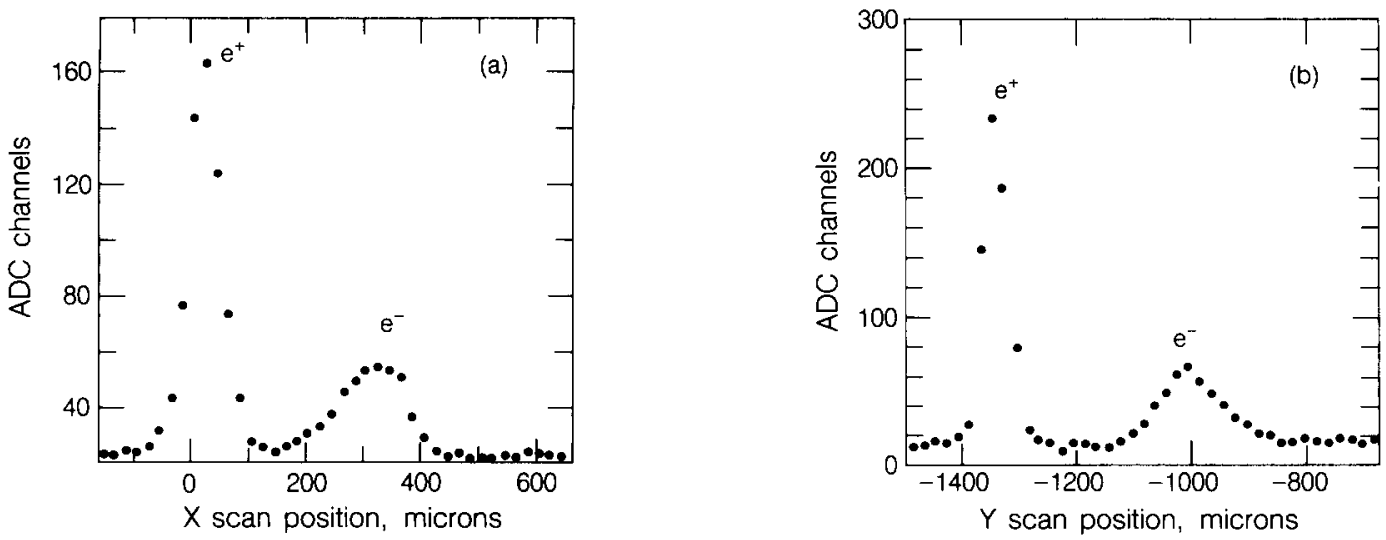

Fig. 10. $X$ and $Y$ scans while both electron and positron beams were present at the IP, but not colliding. These scans were taken with the large fiber. 
impact parameters) with the passing projectile particle. A beam particle produces a secondary electron in a few percent of the collisions, based upon previous reports [3-7]. In the present device, we observe secondary emission efficiencies which depend upon wire diameter and also upon beam particle, $\mathrm{e}^{-}$or $\mathrm{e}^{+}$. It is about $1 \%$ for an $\mathrm{e}^{-}$beam with the $25 \mu \mathrm{m}$ diameter fiber. Based upon only one instance where sufficient data was available, it appears that the efficiency for $\mathrm{e}^{+}$is significantly higher than for $\mathrm{e}^{-}$. A difference in response between $\mathrm{e}^{-}$and $\mathrm{e}^{+}$may well be expected from the effects of the electric field of the beam bunch upon the emitted secondary electrons. Another effect, observed by others but not seen by us is that of signal enhancement by placing a small $(\sim 30 \mathrm{~V})$ negative $\mathrm{dc}$ bias on the wire: as much as a factor of two signal increase has been reported [5]. This is explained as a repulsion from the wire of some very low energy secondary electrons which would otherwise return, due to a negative ambient potential in the neighborhood. In the present case, it seems likely that the intense electric field from the SLC bunches (up to several $\mathrm{MV} / \mathrm{cm}$ ) dominates the local potential so there is little influence from a relatively small $\mathrm{dc}$ bias voltage.

\subsection{Heating of the carbon fibers}

In thin fibers, part of the ionization energy loss escapes in the form of delta rays [8]. The greater part, however, remains to heat the fiber and very simple considerations show that the carbon sublimation point would easily be exceeded in a single SLC pulse fully focussed onto the fiber with design intensity and spot size. At lower intensities, thermal shock may cause failure, and the threshold for this is very uncertain. During the SLC development period discussed here, the beam intensity was always less than $5 \times 10^{9}$ particles per pulse, the transverse rms dimensions were greater than $5 \mu \mathrm{m}$, and all fibers survived. Under these conditions the maximum temperature of the fiber is calculated to be less than $300^{\circ} \mathrm{C}$. Between SLC pulses the fibers cool by conduction primarily, with some radiation cooling at higher temperatures. This should be true up to the full $120 \mathrm{~Hz}$ repetition rate, but for this report the SLC operating frequency was only $5 \mathrm{~Hz}$.

\section{Summary}

A wire scanner making use of secondary emission from very thin carbon fibers has been used successfully in the measurement of beam profiles at the Stanford Linear Collider. Beams as small as $5 \mu \mathrm{m}$ rms have been measured. After proper shielding techniques were implemented, the instrument has proven to be quite reliable and was used routinely in the tuneup of the final focus section of the collider.

\section{Acknowledgements}

Many people have contributed generously their effort and interest to make the wire scanner a successfully operating instrument. We wish to acknowledge in particular Nan Phinney, Tony Gromme and Joanne Bogart for their work on the software, and Dan Wright, for his help on vacuum problems and other installation work. The ideas, assistance and support of Gerry Fischer, Roger Erickson, Ted Fieguth and Philip Bambade were very valuable, and are gratefully acknowledged, as well as other ideas received from others who had also worked on wire scanners, especially R. Jung and L. Evans at CERN, and J. Seeman at SLAC.

\section{References}

[1] SLC Design Handbook, Stanford Linear Accelerator Center, Stanford, CA 94309, USA (December 1984).

[2] J.S. Allen, Phys. Rev. 55 (1939) 336; A.G. Hill et al., Phys. Rev. 55 (1939) 463.

[3] J. Bosser et al., Nucl. Instr. and Meth. A235 (1985) 475.

[4] R. Jung and R.J. Colchester, CERN/LEP-BI/85-160 (1985).

[5] J. Kider and C. Hojvat, Fermilab-Pub-85/176 (1985).

[6] W.T. Weng et al., IEEE Trans. Nucl. Sci. NS-30 (1983) 2331.

[7] R. Chehab et al., LAL/RT/85-05 (Orsay) (1985).

[8] J. Bosser et al., CERN SPS/86-26 (MS) (1986). Our estimate of energy carried away by delta rays is significantly smaller than the $70 \%$ quoted in this reference.

[9] The stage, stepping motor and digital encoder were supplied as a unit by Klinger Scientific Corporation, Richmond Hill, NY 11418, USA. The model used was: UT100$75 \mathrm{PP}$. 